\title{
Cerebral perfusion in the predementia stages of Alzheimer's disease
}

\author{
Maja A. A. Binnewijzend ${ }^{1}$ - Marije R. Benedictus ${ }^{2}$ - Joost P. A. Kuijer ${ }^{3}$. \\ Wiesje M. van der Flier $^{2,4}$ - Charlotte E. Teunissen ${ }^{5} \cdot$ Niels D. Prins $^{2} \cdot$ Mike P. Wattjes $^{1}$. \\ Bart N.M. van Berckel ${ }^{1} \cdot$ Philip Scheltens $^{2} \cdot$ Frederik Barkhof $^{1}$
}

Received: 9 February 2015 /Revised: 24 April 2015 / Accepted: 29 April 2015 / Published online: 5 June 2015

(C) The Author(s) 2015. This article is published with open access at Springerlink.com

\begin{abstract}
Objectives To investigate arterial spin-labelling (ASL) cerebral blood flow (CBF) changes in predementia stages of Alzheimer's disease (AD).

Methods Data were obtained from 177 patients with subjective complaints, mild cognitive impairment and AD from the Amsterdam Dementia Cohort. AD stages were based on diagnosis and cerebrospinal fluid biomarkers amyloid- $\beta(\mathrm{A} \beta)$ and total-tau (tau). Generallinear-models were used to assess relationships between $\mathrm{AD}$ stages and total and regional $\mathrm{CBF}$, correcting for age and sex.

Results Decreasing CBF was related to more advanced $\mathrm{AD}$ stages in all supratentorial regions ( $\mathrm{p}$ for trend $<0.05$ ). Post-
\end{abstract}

Marije R. Benedictus

m.benedictus@vumc.nl

1 Department of Radiology and Nuclear Medicine, Neuroscience Campus Amsterdam, Alzheimercenter Amsterdam, VU University Medical Center, P.O. Box 7057, 1007

MB Amsterdam, The Netherlands

2 Department of Neurology, Neuroscience Campus Amsterdam, Alzheimer Center Amsterdam, VU University Medical Center, P.O. Box 7057, 1007 MB Amsterdam, The Netherlands

3 Department of Physics and Medical Technology, Neuroscience Campus Amsterdam, Alzheimer Center Amsterdam, VU University Medical Center, P.O. Box 7057, 1007 MB Amsterdam, The Netherlands

4 Department of Epidemiology and Biostatistics Neuroscience Campus Amsterdam, Alzheimer Center Amsterdam, VU University Medical Center, P.O. Box 7057, 1007 MB Amsterdam, The Netherlands

5 Department of Clinical Chemistry, Neuroscience Campus Amsterdam, Alzheimer Center Amsterdam, VU University Medical Center, P.O. Box 7057, 1007 MB Amsterdam, The Netherlands hoc testing revealed that $\mathrm{CBF}$ was lower in $\mathrm{AD}$ compared to controls and stage- 1 predementia patients (i.e. abnormal $A \beta$ and normal tau) in temporal and parietal regions, and compared to stage- 2 predementia patients (i.e. abnormal $\mathrm{A} \beta$ and tau) in temporal regions. $\mathrm{CBF}$ values of stage- 2 predementia patients were numerically in between those of stage-1 predementia patients and AD.

Conclusion The continuing decrease of CBF along the continuum of AD indicates the potential of ASL-CBF as a measure for disease progression.

Key Points

- Decreasing CBF relates to more advanced AD stages in all supratentorial regions.

- The reduction of CBF does not reach a bottom level.

- ASL-CBF has potential as a measure for disease progression in $A D$.

Keywords Arterial spin-labelling MRI · Cerebral blood flow/cerebral perfusion · Alzheimer's disease $\cdot$ Prodromal $\mathrm{AD} \cdot$ Disease progression

$\begin{array}{ll}\text { Abbreviations } \\ \text { A } \beta & \text { Amyloid beta } \\ \text { NGMV } & \text { Normalized grey matter volume } \\ \text { PCASL } & \text { Pseudo-continuous aterial spin-labelling } \\ \text { PPC } & \text { Precuneus and posterior cingulated } \\ \text { PVC } & \text { Partial volume corrected } \\ \text { P-tau } & \text { Phosphorylated-tau } \\ \text { SC } & \text { Subjective complaints } \\ \text { T-tau } & \text { Total-tau } \\ \text { WMH } & \text { White matter hyperintensities }\end{array}$




\section{Introduction}

The first pathological signs of Alzheimer's disease (AD), in the form of amyloid- $\beta_{1-42}(A \beta)$ plaque deposition, can be detected up to decades before clinical symptoms first occur [1]. However, these very early pathological signs of AD do not correlate with disease progression in clinical AD [2]. Neuronal and synaptic function measures, such as CSF total-tau (tau) and ${ }^{18} \mathrm{~F}$-fluorodeoxyglucose positron emission tomography (FDG-PET), are thought to start changing later on in the disease process. Moreover, they are thought to relate to $\operatorname{cog}$ nitive performance, and to continue to change along the disease process, which might make them more suitable for monitoring disease progression [2-4].

Arterial spin-labelling (ASL) is a functional magnetic resonance imaging (MRI) technique that measures cerebral blood flow (CBF) by using magnetically labelled arterial blood that flows through the carotid and vertebral arteries as an endogenous contrast medium. Important advantages of ASL are its non-invasiveness and short acquisition time at higher magnetic field strengths, which allows routine clinical application in the workup of dementia when ASL is added to the standard dementia imaging protocol.

Recently, new diagnostic and scientific criteria for clinical $\mathrm{AD}$ and its predementia stages have been introduced by the National Institute on Aging and the Alzheimer's Association (NIA-AA) [5-7]. For scientific purposes, these criteria offer the possibility of subdividing subjects with normal cognition, such as patients with subjective complaints (SC), and patients with mild cognitive impairment (MCI) into different predementia $A D$ stages using $A \beta$ and neuronal injury biomarkers.

In a previous study we showed that ASL discriminated AD patients from controls, and that regional $\mathrm{CBF}$ values of $\mathrm{MCI}$ patients were numerically in between those of controls and $\mathrm{AD}$ patients [8]. In the current study we aimed to further investigate $\mathrm{CBF}$ along the course of $\mathrm{AD}$, by studying $\mathrm{CBF}$ of patients in different predementia stages, based on the NIAAA criteria [5-7].

\section{Methods}

\section{Subjects}

In this cross-sectional study we included 275 patients (107 $\mathrm{AD}$ patients, $64 \mathrm{MCI}$ patients and $104 \mathrm{SC}$ patients) from the memory clinic-based Amsterdam Dementia Cohort. All patients visited our memory clinic between October 2010 and June 2012, underwent brain MRI according to the dementia protocol described below, and had cerebrospinal fluid (CSF) available for analysis of CSF biomarkers. All patients underwent a standard dementia screening that included medical history, physical and neurological examinations, screening laboratory tests, lumbar puncture, neuropsychological testing and brain MRI. Clinical diagnosis was established by consensus in a multidisciplinary team. AD patients met the NINCDS-ADRDA criteria for probable AD [9], and also met the core clinical criteria for probable $\mathrm{AD}$ proposed by the NIA-AA workgroup [6]. MCI patients fulfilled the Petersen criteria $[10,11]$. Patients were considered to have subjective complaints when clinical investigations and neuropsychological test performance were normal. Patients with a history of head trauma or intracranial tumours were excluded. The local Institutional Review Board approved the study. All patients provided written informed consent.

\section{MRI acquisition}

MRI examinations were performed on a $3 \mathrm{~T}$ whole body MR system (SignaHDxt, GE Medical Systems Milwaukee, WI, USA) using an 8-channel head coil. Structural images included a sagittal 3D T1-weighted sequence (IR-FSPGR, echo time $=3.0 \mathrm{~ms}$, repetition time $=7.8 \mathrm{~ms}$, inversion time $=450 \mathrm{~ms}$, flip angle $=12^{\circ}$, matrix $256 \times 256,176$ slices, voxel size $1 \times 0.9 \times 0.9 \mathrm{~mm}$ ) for anatomical information, and a sagittal $3 \mathrm{D}$ fluid attenuated inversion recovery (FLAIR) sequence (CUBE, echo time $=123.6 \mathrm{~ms}$, repetition time $=8000 \mathrm{~ms}$, inversion time $=2351 \mathrm{~ms}$, echo-train length $=230$, acquisition matrix $224 \times 224$, reconstruction matrix $256 \times 256,132$ slices, voxel size $1.2 \times 1 \times 1 \mathrm{~mm}$ ) to determine the severity of white matter hyperintensities (WMH) using the Fazekas scale [12]. Pseudo-continuous ASL (PCASL) [13, 14] perfusion images (3D-FSE acquisition with background suppression, post-label delay $2.0 \mathrm{~s}$, echo time $=9 \mathrm{~ms}$, repetition time $=4.8 \mathrm{~s}$, spiral readout eight arms $\times 512$ samples; $36 \times 5.0 \mathrm{~mm}$ axial slices, $3.2 \times 3.2 \mathrm{~mm}$ in-plane resolution, reconstructed pixel size $1.7 \times 1.7 \mathrm{~mm}$, acquisition time $4 \mathrm{~min}$ ) were calculated using a single-compartment model [15] after the subtraction of labelled from control images. More details are provided in an earlier study by Binnewijzend et al. [8].

\section{Pre-processing and MRI data analysis}

Both T1-weighted and PCASL images were corrected for gradient non-linearities in all three directions. Further data analyses were carried out using FSL (version 4.1; http://www. fmrib.ox.ac.uk/fsl). Pre-processing of T1 images consisted of non-brain tissue removal [16], linear registration to standard space [17] and tissue segmentation [18] yielding partial volume estimates. Gray matter volumes, normalized for subject head size (NGMV), were calculated with the SIENAX software tool [16]. The CBF maps [8] were linearly registered to the brain-extracted $\mathrm{T} 1$ images. The brain mask was used to calculate uncorrected mean whole brain CBF. Partial volume estimates were transformed to the ASL data space and used in 
a regression algorithm [19], using a Gaussian kernel of 9.5$\mathrm{mm}$ full width at half maximum, to create partial volume corrected (PVC) cortical CBF maps. Partial volume estimates were subsequently used as a weighting factor to calculate mean cortical CBF. The MNI152 atlas and the HarvardOxford cortical atlas (both part of FSL) were used to create regions of interest (ROIs) of the frontal, temporal, parietal, precuneus and posterior cingulate (PPC), occipital and cerebellar brain areas, to extract mean regional uncorrected and $\mathrm{PVC}$ cortical CBF values.

\section{CSF analysis}

CSF was obtained by lumbar puncture of the L3/L4 or L4/L5 intervertebral space, using a 25 -gauge needle, and collected via a syringe in 12-ml polypropylene tubes (Sarstedt). A small amount of CSF was used for routine analysis, including total cells (leucocytes and erythrocytes), total protein and glucose. Within 2 hours, CSF samples were centrifuged at $2100 \times g$ for $10 \mathrm{~min}$ at $4^{\circ} \mathrm{C}$ and the supernatant was transferred into a second polyprolylene tube (Sarstedt) and stored at $-20^{\circ} \mathrm{C}$ until $\mathrm{AD}$ biomarker analysis. CSF amyloid- $\beta_{1-42}$ and total-tau were measured with Innotest (Innogenetics) sandwich enzymelinked immunosorbent assay as described previously [20]. The team involved in the CSF analysis was not aware of the clinical diagnosis.

\section{Definition of AD stages}

Patients were divided into AD stages based on the combination of both syndrome diagnosis and CSF biomarker values, considering $\mathrm{A} \beta$ below $550 \mathrm{ng} / \mathrm{L}$, and tau above $375 \mathrm{ng} / \mathrm{L}$ as abnormal (i.e. positive) [20]. For $\mathrm{AD}$, only patients with a high likelihood of having an AD pathophysiology were included. AD patients with normal $\mathrm{A} \beta$ and/or tau values $(\mathrm{n}=$ 41) were excluded. For predementia stages, we collapsed MCI and preclinical stages to overall predementia $\mathrm{AD}$ stages to ensure sufficient power. Only patients fitting in the biomarker stages were included. Therefore, MCI patients with normal $A \beta$ values $(n=42)$ and SC patients with normal $A \beta$ and abnormal tau values $(\mathrm{n}=13)$ were excluded.

Subsequently, MR images of all patients were visually inspected. One AD patient was excluded because registration of the ASL images to T1 images failed, possibly due to suboptimal quality of the ASL images. One other AD patient was excluded because of haemochromatosis.

This resulted in the following four study groups: $80 \mathrm{SC}$ patients with normal CSF A $\beta$ and tau (controls, stage 0), 13 predementia patients (six SC, seven $\mathrm{MCI}$ ) with abnormal CSF $\mathrm{A} \beta$ and normal CSF tau (stage 1), 20 predementia patients (five SC, $15 \mathrm{MCI}$ ) with abnormal CSF $A \beta$ and tau (stage 2) and 64 clinically demented AD patients with abnormal CSF $\mathrm{A} \beta$ and tau (stage 3).

\section{Statistics}

Statistical analyses were performed using IBM SPSS Statistics for Mac (Version 19.0., IBM Corp., Armonk, NY, USA). For continuous measures, differences between groups were assessed using one-way analyses of variance (ANOVA) with post-hoc Bonferroni tests to correct for multiple comparisons. A chi-squared test was used to compare frequency distributions of sex in all study groups, and syndrome diagnosis (i.e. $\mathrm{SC}$ or $\mathrm{MCI}$ ) in the predementia groups. General linear models (GLMs) were used to assess the relationships between $\mathrm{AD}$ stages (entered as continuous measure to assess the doseresponse effect) and total and regional uncorrected and PVC cortical CBF, correcting for the effect of age and sex. Posthoc, univariate GLMs (total CBF) and multivariate GLMs (regional CBF) with Bonferroni tests were performed to compare $\mathrm{CBF}$ values of different $\mathrm{AD}$ stages, correcting for the effect of age and sex (model 1), and additionally for WMH (model 2). Linear regression analyses were performed across groups and within the study groups to assess relationships between CBF (independent variable) and cognition, using MMSE-scores (dependent variable). Age and sex, and additionally $\mathrm{WMH}$, were entered into the model as covariates.

\section{Results}

Demographics, MRI findings and CSF results are presented in Table 1. Stage- 2 predementia patients and stage-3 AD dementia patients were older than controls. Patients with AD dementia had lower MMSE-scores compared to all the other groups. Stage-2 predementia patients and AD dementia patients had more WMH than controls. NGMV was lower in AD dementia patients compared to controls and stage-1 predementia patients, and NGMV was lower in stage-2 predementia patients compared to controls. Finally, by definition, CSF A $\beta$ was lower in all groups compared to controls, and CSF tau was higher in $\mathrm{AD}$ dementia patients and stage- 2 predementia patients compared to controls and stage-1 predementia patients.

\section{Total CBF differences}

With increasing $\mathrm{AD}$ stage, both uncorrected whole brain and PVC cortical CBF decreased ( $\mathrm{p}$ for trend $<0.01$ ) (Table 2). Post-hoc tests showed that AD dementia patients had lower uncorrected whole brain CBF compared to controls and stage-1 predementia patients, and lower PVC cortical CBF compared to controls. There were no differences in $\mathrm{CBF}$ between predementia patients and controls. Additional correction for WMH did not change the group differences. 
Table 1 Patient characteristics, magnetic resonance imaging (MRI) findings and cerebrospinal fluid (CSF) data

Table 2 Region-of-interest based cerebral blood flow (CBF) values

\begin{tabular}{|c|c|c|c|c|}
\hline & \multirow{2}{*}{$\begin{array}{l}\text { Control } \\
\text { Stage } 0\end{array}$} & \multirow{2}{*}{$\begin{array}{l}\text { Predementia } \\
\text { Stage } 1\end{array}$} & \multicolumn{2}{|l|}{$\mathrm{AD}$} \\
\hline & & & Stage 2 & Stage 3 \\
\hline Number & 80 & 13 & 20 & 64 \\
\hline Age $(y)^{\mathrm{a}}$ & $58 \pm 9$ & $64 \pm 7$ & $66 \pm 5^{*}$ & $66 \pm 7 *$ \\
\hline Females, no. (\%) & $31(39)$ & $5(39)$ & $9(45)$ & $34(53)$ \\
\hline Diagnosis (MCI), no. (\%) & - & $7(54)$ & $15(75)$ & - \\
\hline $\mathrm{MMSE}^{\mathrm{a}, \mathrm{b}}$ & $28 \pm 2$ & $28 \pm 2$ & $26 \pm 2$ & $21 \pm 4^{*, \#, \S}$ \\
\hline $\mathrm{WMH}^{\mathrm{a}}$ & $0.6 \pm 0.7$ & $1.2 \pm 0.9$ & $1.2 \pm 0.8^{*}$ & $1.1 \pm 0.8^{*}$ \\
\hline $\operatorname{NGMV}(\mathrm{mL})^{\mathrm{a}}$ & $780 \pm 53$ & $770 \pm 55$ & $733 \pm 63^{*}$ & $711 \pm 46^{*, *}$ \\
\hline $\operatorname{CSF} A \beta_{1-42}(n g / L)^{a}$ & $986 \pm 188$ & $444 \pm 89 *$ & $442 \pm 70^{*}$ & $459 \pm 68^{*}$ \\
\hline $\mathrm{CSF}$ tau $(\mathrm{ng} / \mathrm{L})^{\mathrm{a}}$ & $220 \pm 73$ & $292 \pm 75$ & $782 \pm 370^{*, *}$ & $796 \pm 340^{*, *}$ \\
\hline
\end{tabular}

${ }^{a}$ Data are given as mean \pm standard deviation

${ }^{\mathrm{b}}$ Mini Mental State Examination (MMSE) scores of two controls were excluded because they were obtained by means of an interpreter

Stage 0: A $\beta$-/tau- patients with subjective complaints (SC), Stage 1: $\mathrm{A} \beta+/$ tau- patients with SC and mild cognitive impairment (MCI), Stage 2: A $\beta+/$ tau + patients with SC and $\mathrm{MCI}$, Stage 3: A $\beta+/$ tau + patients with $\mathrm{AD}$ ${ }^{*} \mathrm{p}<0.05$ compared to controls, ${ }^{¥} \mathrm{p}<0.05$ compared to stage- 1 predementia patients, ${ }^{\S} \mathrm{p}<0.05$ compared to stage2 predementia patients

$A D$ Alzheimer's disease, $W M H$ white matter hyperintensities (based on Fazekas-score), $N G M V$ normalized grey matter volume

\begin{tabular}{|c|c|c|c|c|}
\hline & \multirow{2}{*}{$\begin{array}{l}\text { Control } \\
\text { Stage } 0\end{array}$} & \multirow{2}{*}{$\begin{array}{l}\text { Predementia } \\
\text { Stage } 1\end{array}$} & \multicolumn{2}{|l|}{$\mathrm{AD}$} \\
\hline & & & Stage 2 & Stage 3 \\
\hline Uncorrected whole brain $\mathrm{CBF}^{* * *}$ & $32 \pm 5$ & $32 \pm 5$ & $30 \pm 5$ & $28 \pm 5^{\mathrm{a}, \mathrm{b}}$ \\
\hline $\mathrm{PVC}$ cortical $\mathrm{CBF}^{* *}$ & $48 \pm 8$ & $48 \pm 7$ & $45 \pm 8$ & $43 \pm 8^{\mathrm{a}}$ \\
\hline \multicolumn{5}{|l|}{ Regional uncorrected cortical CBF } \\
\hline Frontal* & $22 \pm 5$ & $21 \pm 5$ & $20 \pm 4$ & $19 \pm 4$ \\
\hline Temporal $* * *$ & $26 \pm 5$ & $26 \pm 5$ & $24 \pm 4$ & $21 \pm 4^{\mathrm{a}, \mathrm{b}, \mathrm{c}}$ \\
\hline Parietal ${ }^{* * *}$ & $29 \pm 5$ & $28 \pm 5$ & $26 \pm 5$ & $24 \pm 5^{\mathrm{a}, \mathrm{d}}$ \\
\hline $\mathrm{PPC}^{* * *}$ & $38 \pm 7$ & $37 \pm 7$ & $34 \pm 6$ & $31 \pm 6^{\mathrm{a}, \mathrm{b}}$ \\
\hline Occipital $* * *$ & $35 \pm 7$ & $34 \pm 6$ & $32 \pm 7$ & $29 \pm 7^{\mathrm{a}, \mathrm{d}}$ \\
\hline Cerebellum & $26 \pm 5$ & $26 \pm 6$ & $25 \pm 6$ & $25 \pm 5$ \\
\hline \multicolumn{5}{|l|}{ Regional PVC cortical CBF } \\
\hline Frontal* & $49 \pm 9$ & $49 \pm 8$ & $47 \pm 9$ & $43 \pm 9$ \\
\hline Temporal** & $44 \pm 7$ & $44 \pm 6$ & $42 \pm 8$ & $39 \pm 7^{\mathrm{a}, \mathrm{d}}$ \\
\hline Parietal ${ }^{* * *}$ & $55 \pm 9$ & $55 \pm 9$ & $51 \pm 10$ & $47 \pm 10^{\mathrm{a}, \mathrm{d}}$ \\
\hline $\mathrm{PPC}^{* * *}$ & $63 \pm 11$ & $62 \pm 10$ & $58 \pm 11$ & $55 \pm 11^{\mathrm{a}, \mathrm{d}}$ \\
\hline Occipital** & $55 \pm 9$ & $54 \pm 9$ & $51 \pm 12$ & $49 \pm 10^{\mathrm{a}}$ \\
\hline Cerebellum & $40 \pm 9$ & $41 \pm 0$ & $36 \pm 8$ & $38 \pm 10$ \\
\hline
\end{tabular}

Results are given as mean \pm standard deviation. If analysis of variance was $\mathrm{p}<0.05$ a post-hoc Bonferroni test was performed. Shown results are corrected for age and sex

${ }^{*} \mathrm{p}<0.05, * * \mathrm{p}<0.01$ and $* * * \mathrm{p}<0.001$

${ }^{\mathrm{a}} \mathrm{p}<0.05$ compared to controls

${ }^{\mathrm{b}} \mathrm{p}<0.05$ compared to stage- 1 predementia patients

${ }^{c} \mathrm{p}<0.05$ compared to stage- 2 predementia patients

${ }^{\mathrm{d}} \mathrm{p}<0.1$ compared to stage- 1 predementia patients

Stage 0: A $\beta$-/tau- patients with subjective complaints (SC), Stage 1: $\mathrm{A} \beta+/$ tau- patients with SC and mild cognitive impairment (MCI), Stage 2: A $\beta+/$ tau+ patients with SC and MCI, Stage 3: A $\beta+/$ tau + patients with AD $A D$ Alzheimer's disease, $P P C$ precuneus and posterior cingulated, $P V C$ partial volume corrected 
Fig. 1 Lower regional cerebral blood flow $(\mathrm{CBF})$ is associated with advancing Alzheimer's disease (AD) stage. Bar graphs display mean regional uncorrected $\mathrm{CBF}$ (a) and mean partial volume corrected (PVC) cortical CBF (b) for the different AD stages, per brain region. Error bars represent standard deviations ( $\pm 2 \mathrm{SD})$. Dose-response relationships between $\mathrm{CBF}$ and $\mathrm{AD}$ stage were detected using general-linear models (AD stage entered as a continuous measure), resulting in a $\mathrm{p}$ for trend $<0.05$ in all brain regions except the cerebellum. The most prominent relationships between decreasing $\mathrm{CBF}$ and advancing $\mathrm{AD}$ stage were found in the posterior supratentorial brain regions ( $\mathrm{p}$ for trend $<0.001)$. $P P C$ precuneus and posterior cingulate cortex.

$* p$ for trend $<0.05$,

$* * \mathrm{p}$ for trend $<0.01$,

$* * * \mathrm{p}$ for trend $<0.001$
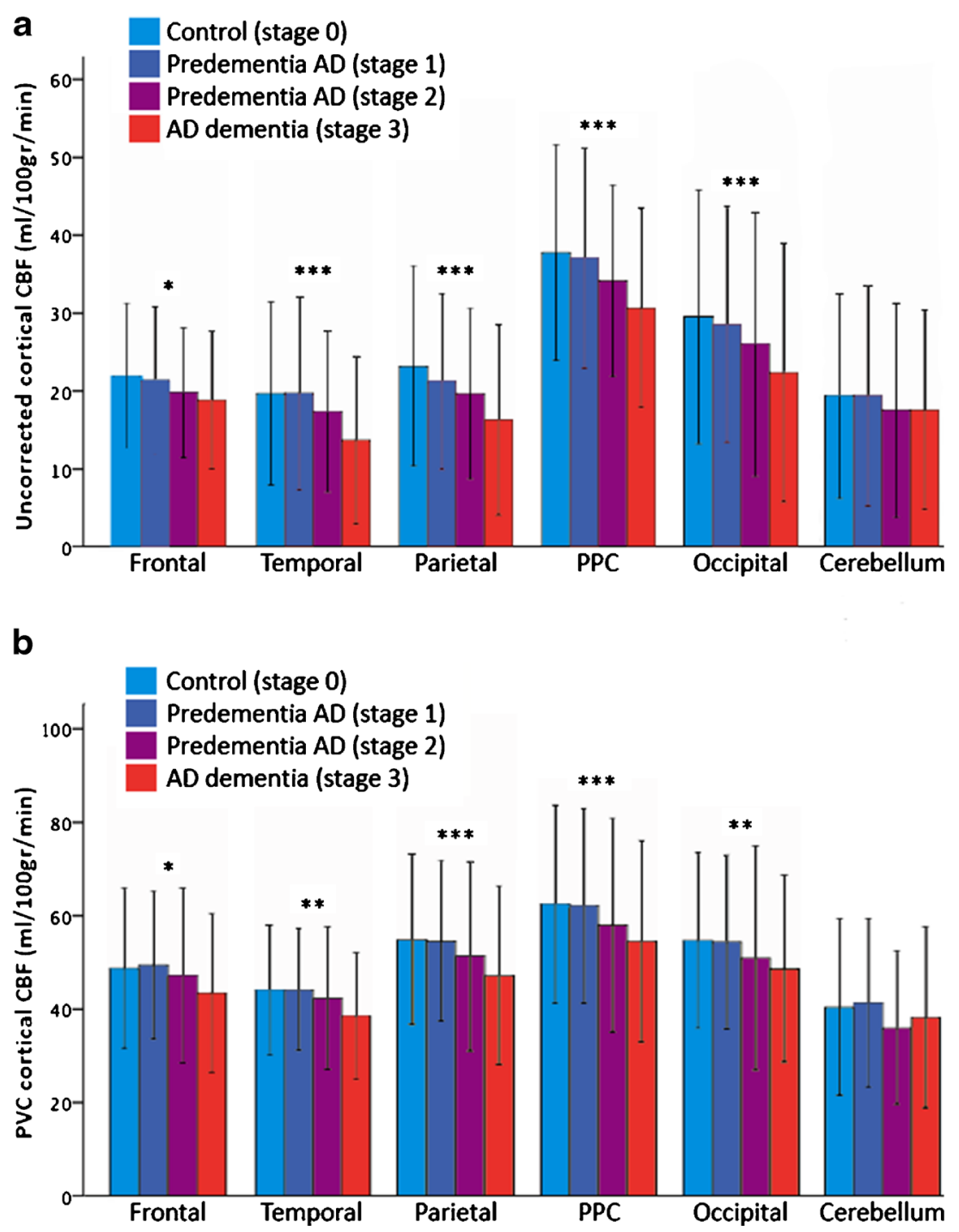

\section{Differences in regional CBF patterns}

As expected, regional PVC cortical CBF values were much higher than regional uncorrected cortical $\mathrm{CBF}$ values, as the latter also contains cerebrospinal fluid and maybe even some edges of white matter (Table 2). A more advanced AD stage was related to lower regional $\mathrm{CBF}$ in all regions except the cerebellum. Relationships were most prominent in the temporal, parietal, PPC and occipital regions for uncorrected cortical $\mathrm{CBF}$, and in the parietal and $\mathrm{PPC}$ regions for PVC cortical CBF ( $p$ for trend $<0.001$; Fig. 1). Posthoc, uncorrected cortical and PVC cortical CBF were lower in temporal, parietal, $\mathrm{PPC}$ and occipital regions in AD dementia patients compared to controls (Table 2). Furthermore, uncorrected cortical CBF was lower in AD dementia patients compared to stage-1 predementia patients in the temporal, parietal (trend), PPC and occipital (trend) regions, and compared to stage- 2 predementia patients in the temporal lobes. AD dementia patients showed a trend towards lower PVC cortical $\mathrm{CBF}$ values compared to stage-1 predementia patients in the temporal, parietal, PPC and occipital regions. No between-group $\mathrm{CBF}$ differences were found in the frontal lobes and the cerebellum, and there were no $\mathrm{CBF}$ differences between stage- 2 predementia patients, stage- 1 predementia patients and controls. Additional correction for WMH did not change the group differences.

\section{Relationship between CBF and cognition}

Across groups, uncorrected whole brain CBF and PVC cortical $\mathrm{CBF}$ were related to cognitive performance in all brain regions, except the cerebellum (Table 3). When we investigated correlations within each stage separately, in AD dementia patients this relationship was seen in the temporal and parietal regions (uncorrected cortical 
Table 3 Relationship between cerebral blood flow (CBF) and cognition (Mini-Mental State Examination scores)

\begin{tabular}{|c|c|c|c|c|c|}
\hline & \multirow[t]{2}{*}{ Across groups } & \multirow{2}{*}{$\begin{array}{l}\text { Control } \\
\text { Stage } 0\end{array}$} & \multirow{2}{*}{$\begin{array}{l}\text { Predementia } \\
\text { Stage } 1\end{array}$} & \multicolumn{2}{|l|}{$\mathrm{AD}$} \\
\hline & & & & Stage 2 & Stage 3 \\
\hline Uncorrected whole brain $\mathrm{CBF}$ & $0.33 * *$ & -0.01 & 0.22 & -0.20 & $0.21^{+}$ \\
\hline PVC cortical CBF & $0.27 * *$ & -0.02 & 0.30 & -0.21 & 0.19 \\
\hline \multicolumn{6}{|c|}{ Regional uncorrected cortical CBF } \\
\hline Frontal & $0.23 *$ & -0.06 & -0.03 & 0.05 & 0.18 \\
\hline Temporal & $0.38^{* *}$ & 0.02 & 0.22 & -0.03 & $0.21^{+}$ \\
\hline Parietal & 0.40 & -0.01 & 0.22 & 0.16 & $0.24^{+}$ \\
\hline PPC & $0.40^{* *}$ & 0.03 & 0.28 & -0.17 & 0.17 \\
\hline Occipital & $0.34 * *$ & 0.12 & $0.25^{\S}$ & $-0.35^{+}$ & 0.18 \\
\hline Cerebellum & 0.09 & -0.05 & 0.24 & -0.30 & -0.04 \\
\hline \multicolumn{6}{|l|}{ Regional PVC cortical CBF } \\
\hline Frontal & $0.24 *$ & -0.07 & 0.18 & -0.21 & $0.23^{+}$ \\
\hline Temporal & $0.31 * *$ & -0.02 & 0.26 & -0.13 & $0.22^{+}$ \\
\hline Parietal & $0.32 * *$ & -0.02 & 0.41 & -0.18 & $0.21^{+}$ \\
\hline PPC & $0.29 * *$ & 0.01 & 0.46 & -0.28 & 0.16 \\
\hline Occipital & $0.23 *$ & 0.04 & 0.44 & -0.30 & 0.11 \\
\hline Cerebellum & 0.08 & -0.04 & 0.13 & -0.05 & 0.02 \\
\hline
\end{tabular}

${ }^{*} \mathrm{p}<0.01,{ }^{* *} \mathrm{p}<0.001,{ }^{+} \mathrm{p}<0.1 ;$ adjustment for age and sex (model 1 )

$\S$ A relationship between cognition and uncorrected CBF in the occipital lobes (standardized beta $0.70 ; \mathrm{p}<0.05$ ) was detected after additional adjustment for white matter hyperintensities (Fazekas-scores; model 2)

Stage 0: A $\beta$-/tau- patients with subjective complaints (SC), Stage 1: A $\beta+/$ tau- patients with SC and mild cognitive impairment (MCI), Stage 2: $A \beta+/$ tau+ patients with $\mathrm{SC}$ and $\mathrm{MCI}$, Stage $3: \mathrm{A} \beta+/$ tau + patients with $\mathrm{AD}$

$A D$ Alzheimer's disease, $P P C$ precuneus and posterior cingulated, $P V C$ partial volume corrected

and PVC cortical CBF; trends), and in the frontal lobes (PVC cortical CBF; trend). Stage-2 predementia patients showed a relationship between uncorrected $\mathrm{CBF}$ and cognition in the occipital lobes (trend). After additional adjustment for WMH (model 2), the essence of the results remained unchanged, with the addition that in stage-1 predementia patients a relationship between cognitive performance and uncorrected $\mathrm{CBF}$ in the occipital lobes reached significance (standardized beta 0.70 , $\mathrm{p}<0.05)$.

\section{Discussion}

The main finding of this study was that lower CBF was observed with more advancing stages of $\mathrm{AD}$ across the spectrum from normal cognition to AD dementia. Furthermore, $\mathrm{CBF}$ was related to cognitive performance across $\mathrm{AD}$ stages and within the stage- $3 \mathrm{AD}$ group.

To date, several ASL studies have detected CBF decreases in $\mathrm{AD}$, most prominently in the bilateral parietal cortex and the precuneus and posterior cingulate cortex [8, 21, 22]. Based on the currently widely supported suggestion that neuronal dysfunction occurs prior to the stage of clinical $\mathrm{AD}$, the aim of this study was to investigate ASL-measured $\mathrm{CBF}$ of $\mathrm{A} \beta$ positive non-demented patients in different disease stages [5, 7]. This resulted in the finding that, compared to $A \beta$ and tau negative controls, ASL-CBF was decreased in AD dementia patients, but not in predementia AD patients. Temporoparietal $\mathrm{CBF}$ was lower in $\mathrm{AD}$ dementia patients than in stage-1 predementia patients, in particular for uncorrected cortical CBF, but to a lesser extent also for PVC cortical CBF. Stage-2 predementia patients showed no decreased CBF compared to controls, but their $\mathrm{CBF}$ did not differ from $\mathrm{AD}$ dementia patients either. In fact, quantitative CBF values of stage-2 predementia patients were numerically in between the $\mathrm{CBF}$ values of stage-1 predementia patients and stage-3 $\mathrm{AD}$ dementia patients. These findings are in accordance with the idea that $\mathrm{CBF}$ alterations, as a sign of neuronal dysfunction, occur further along the disease process than the accumulation of $\mathrm{A} \beta$, but before the stage of clinical dementia.

This is the first study comparing ASL-measured CBF of predementia $\mathrm{AD}$ stages with study groups based on CSF biomarker profiles [5-7]. Previous ASL studies investigating cerebral perfusion in MCI detected parietal and medial temporal hypoperfusion in a less prominent and widespread pattern than the hypoperfusion found in $\mathrm{AD}$ compared to controls [8, 21-26]. However, none of these studies reported amyloid 
status of the MCI patients. This leaves room for the possibility that other $\mathrm{CBF}$-decreasing non-AD pathology caused regional CBF changes in MCI, such as DLB, which has previously been shown to also be related to severe CBF decreases [27, 28]. In our study, we therefore used biomarkers to define our study groups, allowing us to examine changes in CBF across the entire spectrum of AD.

In the current hypothetical biomarker models for AD, FDGPET is considered the main functional neuroimaging tool to assess neuronal injury in terms of hypometabolism in $\mathrm{AD}[3,4$, 29]. Since glucose metabolism and perfusion are closely related, ASL is a potential alternative for the visualization of cerebral and neuronal function [30-32]. However, the relationship between perfusion and metabolism has not yet been thoroughly investigated in the predementia stages of AD. One PET study by Drzezga et al. [33] found lower regional glucose metabolism in $A \beta$-positive healthy controls compared to $A \beta$ negative controls, suggesting a relationship between $A \beta$ and glucose metabolism. This is not consistent with our finding that ASL-measured CBF did not change with the presence of amyloid pathology in a very early stage of AD. Direct comparisons of FDG-PET measured glucose metabolism and ASL-measured cerebral perfusion in predementia AD are needed to clarify whether glucose metabolism and cerebral perfusion are directly related or consecutive processes.

In a previous study we showed that cognitive performance was related to ASL-measured $\mathrm{CBF}$ in $\mathrm{AD}$ [8]. In the current study we also found this relationship within the AD dementia group, albeit in fewer regions and less strong. This discrepancy may be due to the fact that in the current study, $\mathrm{AD}$ patients were selected based on CSF biomarkers in addition to clinical diagnosis, possibly causing less variability in both cognition and $\mathrm{CBF}$ within the $\mathrm{AD}$ dementia group compared to our previous study, in which the $\mathrm{AD}$ population also contained subjects with normal or borderline normal CSF A $\beta$ and tau values. Besides a trend towards a significant relationship between occipital CBF and cognition in the stage-2 predementia group (in the unexpected direction), no relationships between $\mathrm{CBF}$ and cognition were found in the $\mathrm{A} \beta$ positive predementia groups. However, it is not inconceivable that a lack of power contributed to this negative result. After adjustment for $\mathrm{WMH}$, a relationship between uncorrected $\mathrm{CBF}$ in the occipital lobes and cognition did appear within the stage-1 predementia group.

This study has several strengths and limitations. First, the study groups were selected based on both clinical diagnosis and CSF biomarkers. This limited our sample sizes, but at the same time resulted in quite homogeneous study groups, strongly reducing the effect of perfusion changes caused by for example neurodegeneration of non-AD origin. Since the criteria for clinical stages in preclinical and prodromal $\mathrm{AD}$ show strong similarities, and implementing all stages in a sequential manner is not easily done, we chose to reduce the number of study groups by combining preclinical and prodromal patient groups with identical biomarker profiles. A quantitative 3D pseudo-continuous ASL sequence with wholebrain coverage was used to study CBF. Furthermore, both $\mathrm{CBF}$ without correction for partial volume effects (representative for clinical practice) and PVC cortical CBF (actual gray matter $\mathrm{CBF}$ ) were studied. The inclusion of subjects with subjective complaints instead of healthy controls might be considered a limitation. However, controls were only included if they had normal biomarkers, strongly decreasing the likelihood that they would have preclinical AD. Additional potential limitations related to image technique include the fact that ASL scan quality could not be assured completely. Although the perfusion images were visually of good quality, the labelling efficiency was not assessed formally. Furthermore, we did not scan with several delay times to account for differences in travel times between groups. However, in line with recently published recommendations [14], a delay time of $2.0 \mathrm{~s}$ (instead of $1.5 \mathrm{~s}$ ) was used to alleviate the effect of delayed blood arrival in the brain in our study groups [34].

In conclusion, we found lower CBF with advancing NIAAA-based stages of AD. A $\beta$-positive predementia patients with normal CSF tau levels showed no reduction in ASLmeasured $\mathrm{CBF}$, endorsing the idea that $\mathrm{CBF}$ changes are not directly related to $A \beta$ deposition. Furthermore, the continuing decrease along the continuum of $\mathrm{AD}$ shows that the reduction of CBF does not reach a bottom level, but is associated with decline severity, suggesting that ASL-measured CBF may be used as a measure for disease progression.

Acknowledgments The authors thank Ajit Shankaranarayanan of GE Healthcare for providing the 3D pseudo-continuous ASL sequence that was used to obtain data for this paper.

The scientific guarantor of this publication is Frederik Barkhof. The authors of this manuscript declare relationships with the following companies: M.A.A. Binnewijzend, M.R. Benedictus J.P.A. Kuijer and W.M. van der Flier report no disclosures. C.E. Teunissen serves on the advisory board of Innogenetics and Roche. She has received speaker honorarium on a symposium organised by Teva. N.D. Prins serves on the advisory board of Boehringer Ingelheim and Envivo. He has been a speaker at symposia organised by Janssen and Novartis. NDP has a senior fellowship at the Alzheimer Center of the VU University Medical Center Amsterdam, partly supported by Vereniging AEGON and receives research support from the Brain Foundation of The Netherlands and Alzheimer Nederland. NDP receives no personal compensation for the activities mentioned above. M.P. Wattjes serves as a consultant for Biogen-Idec. Dr. Wattjes receives research support from Biogen Idec, Bayer Healthcare, Roche and Janssen Cilag. Dr. Wattjes serves as an editorial board member of European Radiology. B.N.M. van Berckel receives research support from the Alzheimer Assistance Foundation, the Center for Translational Molecular Imaging, the Alzheimer Association, De Hersenstichting Nederland and the Internationale Stichting Alzheimer Onderzoek. Dr. van Berckel receives no personal compensation from these organizations. P. Scheltens receives grant support (for the institution; the Alzheimer Center of the VU University Medical Center Amsterdam, see also below) from GE Healthcare, Danone Research and MERCK. In the past 2 years he has received speaker's fees (paid to the institution) from Lilly, GE Healthcare, Lundbeck, Danone and Jansen 
AI-Pfizer. He is co-editor-in-chief of Alzheimer Research \& Therapy. F. Barkhof serves on the editorial boards of Brain, European Radiology, Radiology, Multiple Sclerosis and Neuroradiology and serves as a consultant for Bayer-Schering Pharma, Sanofi-Aventis, Biogen-Idec, TEVA, Synthon BV, Merck-Serono, Jansen Research, Novartis and Roche. Dr. Barkhof receives research support from the Dutch MS Society (EU-FP7). Dr. Barkhof has received consulting fees or honoraria for the consultancy mentioned above. Dr. Barkhof has received speaker's fees from the Serono Symposia Foundation.

The study was supported by the Alzheimer Center of the VU University Medical Center Amsterdam, The Netherlands. Research from the Alzheimer Center of the VU University Medical Center Amsterdam is part of the neurodegeneration research programme of the Neuroscience Campus Amsterdam. The Alzheimer Center of the VU University Medical Center Amsterdam is supported by Alzheimer Nederland and Stichting VUmc funds. The clinical database structure was developed with funding from Stichting Dioraphte.

One of the authors has significant statistical expertise (WM van der Flier). Institutional Review Board approval was obtained. Written informed consent was obtained from all subjects (patients) in this study. Some study subjects or cohorts have been previously reported in:

a) Binnewijzend MA, Kuijer JP, Benedictus MR, van der Flier WM, Wink AM, Wattjes MP et al. Cerebral blood flow measured with 3D pseudocontinuous arterial spin-labeling $\mathrm{mr}$ imaging in alzheimer disease and mild cognitive impairment: a marker for disease severity. Radiology 2013;267:221-30.

b) Binnewijzend MA, Kuijer JP, van der Flier WM, Benedictus MR, Moller CM, Pijnenburg YA et al. Distinct perfusion patterns in Alzheimer's disease, frontotemporal dementia and dementia with Lewy bodies. Eur Radiol 2014;24:2326-33.

Methodology: retrospective, cross-sectional study, performed at one institution.

Open Access This article is distributed under the terms of the Creative Commons Attribution-NonCommercial 4.0 International License (http://creativecommons.org/licenses/by-nc/4.0/), which permits any noncommercial use, distribution, and reproduction in any medium, provided you give appropriate credit to the original author(s) and the source, provide a link to the Creative Commons license, and indicate if changes were made.

\section{References}

1. Jack CR Jr, Lowe VJ, Weigand SD, Wiste HJ, Senjem ML, Knopman DS et al (2009) Serial PIB and MRI in normal, mild cognitive impairment and Alzheimer's disease: implications for sequence of pathological events in Alzheimer's disease. Brain 132: 1355-1365

2. Jagust WJ, Landau SM, Shaw LM, Trojanowski JQ, Koeppe RA, Reiman EM et al (2009) Relationships between biomarkers in aging and dementia. Neurology 73:1193-1199

3. Jack CR, Knopman DS, Jagust WJ, Shaw LM, Aisen PS, Weiner MW et al (2010) Hypothetical model of dynamic biomarkers of the Alzheimer's pathological cascade. Lancet Neurol 9:119-128

4. Jack CR Jr, Knopman DS, Jagust WJ, Petersen RC, Weiner MW, Aisen PS et al (2013) Tracking pathophysiological processes in Alzheimer's disease: an updated hypothetical model of dynamic biomarkers. Lancet Neurol 12:207-216

5. Albert MS, DeKosky ST, Dickson D, Dubois B, Feldman HH, Fox $\mathrm{NC}$ et al (2011) The diagnosis of mild cognitive impairment due to Alzheimer's disease: recommendations from the National Institute on Aging-Alzheimer's Association workgroups on diagnostic guidelines for Alzheimer's disease. Alzheimers Dement 7:270-279
6. McKhann GM, Knopman DS, Chertkow H, Hyman BT, Jack CR Jr, Kawas CH et al (2011) The diagnosis of dementia due to Alzheimer's disease: recommendations from the National Institute on Aging-Alzheimer's Association workgroups on diagnostic guidelines for Alzheimer's disease. Alzheimers Dement 7:263-269

7. Sperling RA, Aisen PS, Beckett LA, Bennett DA, Craft S, Fagan $A M$ et al (2011) Toward defining the preclinical stages of Alzheimer's disease: recommendations from the National Institute on Aging-Alzheimer's Association workgroups on diagnostic guidelines for Alzheimer's disease. Alzheimers Dement 7:280-292

8. Binnewijzend MA, Kuijer JP, Benedictus MR, van der Flier WM, Wink AM, Wattjes MP et al (2013) Cerebral blood flow measured with 3D pseudocontinuous arterial spin-labeling MR imaging in Alzheimer disease and mild cognitive impairment: a marker for disease severity. Radiology 267:221-230

9. McKhann G, Drachman D, Folstein M, Katzman R, Price D, Stadlan EM (1984) Clinical diagnosis of Alzheimer's disease: report of the NINCDS-ADRDA Work Group under the auspices of Department of Health and Human Services Task Force on Alzheimer's Disease. Neurology 34:939-944

10. Petersen RC, Stevens JC, Ganguli M, Tangalos EG, Cummings JL, DeKosky ST (2001) Practice parameter: early detection of dementia: mild cognitive impairment (an evidence-based review). Report of the Quality Standards Subcommittee of the American Academy of Neurology. Neurology 56:1133-1142

11. Petersen RC (2004) Mild cognitive impairment as a diagnostic entity. J Intern Med 256:183-194

12. Fazekas F, Chawluk JB, Alavi A, Hurtig HI, Zimmerman RA (1987) MR signal abnormalities at $1.5 \mathrm{~T}$ in Alzheimer's dementia and normal aging. AJR Am J Roentgenol 149:351-356

13. Dai W, Garcia D, de BC, Alsop DC (2008) Continuous flow-driven inversion for arterial spin labeling using pulsed radio frequency and gradient fields. Magn Reson Med 60:1488-1497

14. Alsop DC, Detre JA, Golay X, Gunther M, Hendrikse J, Hernandez-Garcia L et al (2014) Recommended implementation of arterial spin-labeled perfusion MRI for clinical applications: A consensus of the ISMRM perfusion study group and the European consortium for ASL in dementia. Magn Reson Med. doi:10.1002/ mrm. 25197

15. Buxton RB, Frank LR, Wong EC, Siewert B, Warach S, Edelman RR (1998) A general kinetic model for quantitative perfusion imaging with arterial spin labeling. Magn Reson Med 40:383-396

16. Smith SM (2002) Fast robust automated brain extraction. Hum Brain Mapp 17:143-155

17. Jenkinson M, Smith S (2001) A global optimisation method for robust affine registration of brain images. Med Image Anal 5: $143-156$

18. Zhang Y, Brady M, Smith S (2001) Segmentation of brain MR images through a hidden Markov random field model and the expectation-maximization algorithm. IEEE Trans Med Imaging 20:45-57

19. Asllani I, Borogovac A, Brown TR (2008) Regression algorithm correcting for partial volume effects in arterial spin labeling MRI. Magn Reson Med 60:1362-1371

20. Mulder C, Verwey NA, van der Flier WM, Bouwman FH, Kok A, van Elk EJ et al (2010) Amyloid-beta(1-42), total tau, and phosphorylated tau as cerebrospinal fluid biomarkers for the diagnosis of Alzheimer disease. Clin Chem 56:248-253

21. Dai W, Lopez OL, Carmichael OT, Becker JT, Kuller LH, Gach HM (2009) Mild cognitive impairment and alzheimer disease: patterns of altered cerebral blood flow at MR imaging. Radiology 250: $856-866$

22. Johnson NA, Jahng GH, Weiner MW, Miller BL, Chui HC, Jagust WJ et al (2005) Pattern of cerebral hypoperfusion in Alzheimer disease and mild cognitive impairment measured with arterial 
spin-labeling MR imaging: initial experience. Radiology 234:851859

23. Bangen KJ, Restom K, Liu TT, Wierenga CE, Jak AJ, Salmon DP et al (2012) Assessment of Alzheimer's disease risk with functional magnetic resonance imaging: an arterial spin labeling study. J Alzheimers Dis 31(Suppl 3):S59-S74

24. Chao LL, Buckley ST, Kornak J, Schuff N, Madison C, Yaffe K et al (2010) ASL perfusion MRI predicts cognitive decline and conversion from MCI to dementia. Alzheimer Dis Assoc Disord 24:19-27

25. Fleisher AS, Podraza KM, Bangen KJ, Taylor C, Sherzai A, Sidhar K et al (2009) Cerebral perfusion and oxygenation differences in Alzheimer's disease risk. Neurobiol Aging 30:1737-1748

26. Kim SM, Kim MJ, Rhee HY, Ryu CW, Kim EJ, Petersen ET et al (2013) Regional cerebral perfusion in patients with Alzheimer's disease and mild cognitive impairment: effect of APOE epsilon4 allele. Neuroradiology 55:25-34

27. Binnewijzend MA, Kuijer JP, van der Flier WM, Benedictus MR, Moller CM, Pijnenburg YA et al (2014) Distinct perfusion patterns in Alzheimer's disease, frontotemporal dementia and dementia with Lewy bodies. Eur Radiol 24:2326-2333

28. Fong TG, Inouye SK, Dai W, Press DZ, Alsop DC (2011) Association cortex hypoperfusion in mild dementia with Lewy bodies: a potential indicator of cholinergic dysfunction? Brain Imaging Behav 5:25-35

29. Frisoni GB, Fox NC, Jack CR Jr, Scheltens P, Thompson PM (2010) The clinical use of structural MRI in Alzheimer disease. Nat Rev Neurol 6:67-77

30. Chen Y, Wolk DA, Reddin JS, Korczykowski M, Martinez PM, Musiek ES et al (2011) Voxel-level comparison of arterial spinlabeled perfusion MRI and FDG-PET in Alzheimer disease. Neurology 77:1977-1985

31. Jueptner M, Weiller C (1995) Review: does measurement of regional cerebral blood flow reflect synaptic activity? Implications for PET and fMRI. Neuroimage 2:148-156

32. Musiek ES, Chen Y, Korczykowski M, Saboury B, Martinez PM, Reddin JS et al (2012) Direct comparison of fluorodeoxyglucose positron emission tomography and arterial spin labeling magnetic resonance imaging in Alzheimer's disease. Alzheimers Dement 8: $51-59$

33. Grimmer T, Faust M, Auer F, Alexopoulos P, Forstl H, Henriksen $G$ et al (2012) White matter hyperintensities predict amyloid increase in Alzheimer's disease. Neurobiol Aging 33:2766-2773

34. Campbell AM, Beaulieu C (2006) Pulsed arterial spin labeling parameter optimization for an elderly population. J Magn Reson Imaging 23:398-403 